\title{
AUTOMORPHISM GROUPS OF POSITIVE ENTROPY ON PROJECTIVE THREEFOLDS
}

\author{
FREDERIC CAMPANA, FEI WANG, AND DE-QI ZHANG
}

\begin{abstract}
We prove two results about the natural representation of a group $G$ of automorphisms of a normal projective threefold $X$ on its second cohomology. We show that if $X$ is minimal then $G$, modulo a normal subgroup of null entropy, is embedded as a Zariski-dense subset in a semi-simple real linear algebraic group of real rank $\leq 2$. Next, we show that $X$ is a complex torus if the image of $G$ is an almost abelian group of positive rank and the kernel is infinite, unless $X$ is equivariantly non-trivially fibred.
\end{abstract}

\section{INTRODUCTION}

Let $X$ be a compact Kähler manifold. For an automorphism $g \in \operatorname{Aut}(X)$, its (topological) entropy $h(g)=\log \rho(g)$ is defined as the logarithm of the spectral radius $\rho(g)$ of the pullback action $g^{*}$ on the total cohomology group of $X$, i.e.,

$$
\rho(g):=\max \left\{|\lambda| ; \lambda \text { is an eigenvalue of } g^{*} \mid \oplus_{i \geq 0} H^{i}(X, \mathbb{C})\right\} .
$$

By the fundamental result of Gromov and Yomdin, the above definition is equivalent to the original dynamical definition of entropy (cf. [11], [21]).

An element $g \in \operatorname{Aut}(X)$ is of null entropy if its (topological) entropy $h(g)$ equals 0 . For a subgroup $G$ of $\operatorname{Aut}(X)$, we define the null subset of $G$ as

$$
N(G):=\{g \in G \mid g \text { is of null entropy, i.e., } h(g)=0\}
$$

which may not be a subgroup. A group $G \leq \operatorname{Aut}(X)$ is of null entropy if every $g \in G$ is of null entropy, i.e., if $G$ equals $N(G)$.

By the classification of surfaces, a complex surface $S$ has some $g \in \operatorname{Aut}(S)$ of positive entropy only if $S$ is bimeromorphic to a rational surface, complex torus, $K 3$ surface or Enriques surface (cf. [5]). See [24] for a similar phenomenon in higher dimensions.

Recall that a normal projective variety $X$ is minimal if it has at worst terminal singularities and the canonical divisor $K_{X}$ is nef (cf. [12, Definition 2.34]). Let $\mathrm{NS}(X)$ be the Neron-Severi group and $\mathrm{NS}_{\mathbb{C}}(X):=\mathrm{NS}(X) \otimes \mathbb{C}$. For a subgroup $G$ of $\operatorname{Aut}(X)$, let $\bar{G} \subseteq \mathrm{GL}\left(\mathrm{NS}_{\mathbb{C}}(X)\right)$ be the Zariski-closure of the action of $G$ on $\mathrm{NS}_{\mathbb{C}}(X)$ (simply denoted by $\left.G \mid \mathrm{NS}_{\mathbb{C}}(X)\right)$, and let $R(\bar{G})$ be its solvable radical, both of which are defined

2000 Mathematics Subject Classification. 32H50, 14J50, 32M05, 37B40 .

Key words and phrases. automorphism, iteration, complex dynamics, topological entropy. 
over $\mathbb{Q}$ (cf. [14, ChI; 0.11, 0.23]). We have a natural composition of homomorphisms: $\iota: G \rightarrow G \mid \mathrm{NS}_{\mathbb{C}}(X) \rightarrow \bar{G}$. Denote by

$$
R(G):=\iota^{-1}(\iota(G) \cap R(\bar{G})) \triangleleft G .
$$

Theorem 1.1. Let $X$ be a 3-dimensional minimal projective variety and $G \leq \operatorname{Aut}(X)$ a subgroup such that $G \mid \mathrm{NS}_{\mathbb{C}}(X)$ is not virtually solvable. Then $R(G) \mid \mathrm{NS}_{\mathbb{C}}(X)$ is virtually unipotent. Replacing $G$ by a suitable finite-index subgroup, $G / R(G)$ is embedded as a Zariski-dense subgroup in $H:=\bar{G} / R(\bar{G})$ so that $H(\mathbb{R})$ is a semi-simple real linear algebraic group and is either of real rank 1 (cf. [14, 0.25]) or locally isomorphic to $\mathrm{SL}_{3}(\mathbb{R})$ or $\mathrm{SL}_{3}(\mathbb{C})$ (where locally isomorphic means: having isomorphic Lie algebras).

The key step of the proof is Theorem 4.2 of which part (1) is a consequence of [7, Theorem 5.1] in which the authors have determined the actions of irreducible lattices in semi-simple real Lie groups of higher rank on threefolds.

For a subgroup $G$ of $\operatorname{Aut}(X)$, the pair $(X, G)$ is non-strongly-primitive, if there are $X^{\prime}$ bimeromorphic to $X$, a finite-index subgroup $G_{1}$ of $G$ and a holomorphic map $X^{\prime} \rightarrow Y$ with $0<\operatorname{dim} Y<\operatorname{dim} X$, such that the induced bimeromorphic action of $G_{1}$ on $X^{\prime}$ is biholomorphic and descends to an action on $Y$ with $X^{\prime} \rightarrow Y$ being $G_{1}$-equivariant. $(X, G)$ is strongly primitive if it is not non-strongly-primitive.

Our second main result is Theorem 1.2 (being generalized to higher dimensions in [9]).

Theorem 1.2. Let $X$ be a 3 -dimensional normal projective variety with only $\mathbb{Q}$-factorial terminal singularities, and $G \leq \operatorname{Aut}(X)$ a subgroup such that $G_{0}:=G \cap \operatorname{Aut}_{0}(X)$ is infinite and the quotient group $G / G_{0}$ is an almost abelian group of positive rank (cf. 2.1 for the terminology). Suppose that the pair $(X, G)$ is strongly primitive. Then $X$ is a complex 3-torus and $G_{0}$ is Zariski-dense in $\operatorname{Aut}_{0}(X)$.

We remark that the almost abelian condition (as defined in 2.1) on $G / G_{0}$ is used to show that the extremal rays on $X$ are $G$-periodic (cf. [25, Theorem 2.13, or Appendix]).

Corollary 1.3. Let $X$ be a 3 -dimensional normal projective variety with only $\mathbb{Q}$-factorial rational singularities, and $G \leq \operatorname{Aut}(X)$ a subgroup of null entropy such that $G \mid \mathrm{NS}_{\mathbb{C}}(X)$ is almost abelian of positive rank. Assume that $(X, G)$ is strongly primitive. Then, $\operatorname{Aut}_{0}(X)=\left\{\operatorname{id}_{X}\right\}$ and $h^{1}\left(X, \mathcal{O}_{X}\right)=0$.

We do not have any example satisfying all the hypotheses of Corollary 1.3.

Let $\tau$ be a primitive cubic or quartic root of $1, E:=\mathbb{C} /(\mathbb{Z}+\mathbb{Z} \tau)$ and $X:=E^{n} /\langle\operatorname{diag}[\tau, \ldots, \tau]\rangle$ (cf. [17, Thm (0.3)], [26, Ex 1.7]). Take some $g$ in $\mathrm{SL}_{n}(\mathbb{Z})$ such that $g$ acts on $X$ as an 
automorphism of infinite order and null entropy. When $n=3$, the group $G:=\langle g\rangle$ satisfies the hypotheses of Corollary 1.3. except the strong primitivity which seems hard to verify. The action of $\langle g\rangle$ on $E^{n}$ is not strongly primitive (cf. Proof of Cor. 3.8).

Remark 1.4. (1) In Theorem 1.1, suppose that $c_{1}(X) \neq 0$. Then the Iitaka fibration $X \rightarrow Y$ is $G$-equivariant, and also non-trivial by the abundance theorem or the classification of surfaces. Replacing $G$ by a subgroup of finite index, we may assume that the induced action of $G$ on $Y$ is trivial (cf. [20, Theorem 14.10]), so $G$ acts faithfully on a general fibre $S$ and the group $G \mid S$ is not of null entropy (since the same holds for $G \mid X$; see Theorem 2.2). Since $K_{S}=K_{X} \mid S \sim_{\mathbb{Q}} 0$, our $S$ is a complex 2-torus, $K 3$ or Enriques surface. So there is a homomorphism $G|X=G| S \rightarrow \mathrm{SO}(1, \rho(S)-1) \leq \operatorname{SL}\left(\operatorname{NS}_{\mathbb{R}}(S)\right)(G$ being replaced by a subgroup of index $\leq 2$ ) with kernel virtually contained in $\operatorname{Aut}_{0}(S)$ and the Picard number $\rho(S) \leq 20$.

(2) In Theorem 1.2, the strong primitivity assumption on $(X, G)$ is necessary by considering $X=S \times T$ and $G=\langle g\rangle \times \operatorname{Aut}_{0}(T)$ where $g$ is of positive entropy on a $K 3$ surface $S$, and $T$ a homogeneous curve ( $\mathbb{P}^{1}$ or elliptic).

(3) The projectivity of $X$ in Theorem 1.1 is used in applying the characterization of a quotient of an abelian threefold (cf. [18]). The projectivity of $X$ in Theorem 1.2 is used in running the minimal model program (only for uniruled varieties).

The following Theorem [1.5 is a direct consequence of [23, Theorem 1.1]; see also the discussion in [6, §6]. It extends the classical Tits alternative [19, Theorem 1].

A compact Kähler manifold $X$ is ruled if it is bimeromorphic to a manifold with a $\mathbb{P}^{1}$-fibration. By a result of Matsumura, $X$ is ruled if $\operatorname{Aut}_{0}(X)$ is not a compact torus (cf. [10, Proposition 5.10]). When $X$ is a compact complex Kähler manifold (or a normal projective variety), set $L:=H^{2}(X, \mathbb{Z}) /($ torsion) (resp. $L:=\mathrm{NS}(X) /($ torsion $)$ ), $L_{\mathbb{R}}:=L \otimes_{\mathbb{Z}} \mathbb{R}$, and $L_{\mathbb{C}}:=L \otimes_{\mathbb{Z}} \mathbb{C}$.

Theorem 1.5. Let $X$ be a compact Kähler (resp. projective) manifold of dimension $n$ and $G \leq \operatorname{Aut}(X)$ a subgroup. Then one of the following properties holds.

(1) $G \mid L_{\mathbb{C}} \geq \mathbb{Z} * \mathbb{Z}$ (the non-abelian free group of rank two), and hence $G \geq \mathbb{Z} * \mathbb{Z}$.

(2) $G \mid L_{\mathbb{C}}$ is virtually solvable and $G \geq K \cap L\left(\operatorname{Aut}_{0}(X)\right) \geq \mathbb{Z} * \mathbb{Z}$ where $L\left(\operatorname{Aut}_{0}(X)\right)$ is the linear part of $\operatorname{Aut}_{0}(X)$ (cf. [10, Definition 3.1, p. 240]) and $K=\operatorname{Ker}(G \rightarrow$ $\mathrm{GL}\left(L_{\mathbb{C}}\right)$ ), so $X$ is ruled (cf. [10, Proposition 5.10]).

(3) There is a finite-index solvable subgroup $G_{1}$ of $G$ such that the null subset $N\left(G_{1}\right)$ of $G_{1}$ is a normal subgroup of $G_{1}$ and $G_{1} / N\left(G_{1}\right) \cong \mathbb{Z}^{\oplus r}$ for some $r \leq n-1$.

In particular, either $G \geq \mathbb{Z} * \mathbb{Z}$ or $G$ is virtually solvable. In Cases (2) and (3) above, $G \mid L_{\mathbb{C}}$ is finitely generated. 
Acknowledgement. We are very grateful to the referee for suggesting the implication “Theorem 4.2 $\Rightarrow$ Theorem [1.1' and giving its proof when $N(G)=1$, as well as lots of constructive suggestions. The last-named author is supported by an ARF of NUS.

\section{Entropy and Algebraic group action}

In this section, we shall recall some definitions and technical results needed in the proofs and establish some easy consequences or already known facts.

2.1. Terminology and notation are as in [12]. Below are some more conventions.

Let $X$ be a compact complex Kähler manifold (resp. a normal projective variety). As in the introduction, set $L:=H^{2}(X, \mathbb{Z}) /\left(\right.$ torsion) $($ resp. $L:=\mathrm{NS}(X) /($ torsion $)), L_{\mathbb{R}}:=$ $L \otimes_{\mathbb{Z}} \mathbb{R}$, and $L_{\mathbb{C}}:=L \otimes_{\mathbb{Z}} \mathbb{C}$. Let $\overline{P(X)}$ be the closure of the Kähler cone (resp. the nef cone $\operatorname{Nef}(X)$, i.e., the closure of the ample cone) of $X$. Elements in $\overline{P(X)}$ are called $n e f$.

For $g \in \operatorname{Aut}(X)$, let

$$
d_{1}(g):=\max \left\{|\lambda| ; \lambda \text { is an eigenvalue of } g^{*} \mid H^{1,1}(X)\right\}
$$

be the first dynamical degree of $g$ (cf. [8, §2.2]). By the generalization of Perron-Frobenius theorem (cf. [3]) applied to $\overline{P(X)}$, for every $g \in \operatorname{Aut}(X)$, there is a nonzero nef class $L_{g}$ (not unique) such that

$$
g^{*} L_{g}=d_{1}(g) L_{g}
$$

We remark that $g$ is of null entropy if and only if so is $g^{-1}$; if this is the case, then for every non-trivial $(1,1)$-class $M$ with $g^{*} M=\lambda M$, we have $|\lambda|=1$.

$G \mid Y$ denotes a naturally (from the context) induced action of $G$ on $Y$. A subvariety $Z \subset X$ is $G$-periodic if $Z$ is stabilized by a finite-index subgroup of $G$. For a complex torus $X$ (as a variety), we have $\operatorname{Aut}_{\text {variety }}(X)=T \rtimes \operatorname{Aut}_{\text {group }}(X)$ where $T=\operatorname{Aut}_{0}(X)$ $(\cong X)$ consists of all the translations of $X$ and $\operatorname{Aut}_{\text {group }}(X)$ is the group of bijective homomorphisms of $X$ (as a torus).

A group $G$ is virtually unipotent (resp. virtually abelian, or virtually abelian of rank $r$ ) if a finite-index subgroup $G_{1}$ of $G$ is unipotent (resp. abelian, or isomorphic to $\mathbb{Z}^{\oplus r}$ ). A group $G$ is virtually solvable (resp. almost abelian, or almost abelian of finite rank $r$, cf. definition after [16, Thm 1.2]) if it has a finite-index subgroup $G_{1}$ and an exact sequence

$$
1 \rightarrow H \rightarrow G_{1} \rightarrow Q \rightarrow 1
$$

such that $H$ is finite, and $Q$ is solvable (resp. abelian, or isomorphic to $\mathbb{Z}^{\oplus r}$ ); by Lemma 2.4, $G$ is almost abelian of finite rank $r$ if and only if $G$ is virtually abelian of rank $r$; replacing $G_{1}$ by a finite-index subgroup, we may assume that the conjugation action of $G_{1}$ (and hence of $H$ ) on $H$ is trivial; so in the above definition of virtually solvable group, we may also assume $H=1$, so that our definition here coincides with the usual definition. 
Theorem 2.2 below follows from Oguiso [16, Lemma 2.5] and Tits [19, Theorem 1].

Theorem 2.2. Let $X$ be a compact Kähler (or projective) manifold of dimension $n$ and $G$ a subgroup of $\operatorname{Aut}(X)$. Then we have:

(1) Suppose that $G$ is of null entropy. Then $G \mid L_{\mathbb{C}}$ is virtually unipotent and hence virtually solvable ( $c f$. 2.1 for notation). Moreover, $G \mid L_{\mathbb{C}}$ is finitely generated.

(2) Suppose that $G \mid L_{\mathbb{C}} \geq \mathbb{Z} * \mathbb{Z}$. Then $G$ contains an element of positive entropy.

Let $X$ be a compact Kähler (resp. projective) manifold of dimension $n$. A sequence $0 \neq L_{1} \cdots L_{k} \in H^{k, k}(X)(1 \leq k<n)$ is quasi-nef if it is inductively obtained in the following way: first $L_{1} \in \overline{P(X)}$; once $L_{1} \cdots L_{j-1} \in H^{j-1, j-1}(X)$ is defined, we define

$$
L_{1} \cdots L_{j}=\lim _{t \rightarrow \infty} L_{1} \cdots L_{j-1} \cdot M_{t}
$$

for some $M_{t} \in \overline{P(X)}$ (cf. [23, $\left.\S 2.2\right]$ ). We remark that for $j \geq 2$, the $L_{j}$ which appears in the construction of the $(j, j)$-class $L_{1} \cdots L_{j}$, may not belong to $\overline{P(X)}$. A group $G \leq \operatorname{Aut}(X)$ is polarized by the quasi-nef sequence $L_{1} \cdots L_{k}(1 \leq k<n)$ if

$$
g^{*}\left(L_{1} \cdots L_{k}\right)=\chi_{1}(g) \cdots \chi_{k}(g)\left(L_{1} \cdots L_{k}\right)
$$

for some characters $\chi_{j}: G \rightarrow\left(\mathbb{R}_{>0}, \times\right)$.

Theorem 2.3 gives criteria of virtual solvability, with (3) proved in [23, Theorem 1.2].

Theorem 2.3. Let $X$ be a compact Kähler (resp. projective) manifold of dimension $n$ and $G$ a subgroup of $\operatorname{Aut}(X)$. Then we have (cf. 2.1 for notation of $\left.L_{\mathbb{C}}\right)$ :

(1) Suppose that $G \mid L_{\mathbb{C}}$ is virtually solvable and its Zariski-closure in $\operatorname{GL}\left(L_{\mathbb{C}}\right)$ is connected. Then $G$ is polarized by a quasi-nef sequence $L_{1} \cdots L_{k}(1 \leq k<n)$.

(2) Conversely, suppose that $G$ is polarized by a quasi-nef sequence $L_{1} \cdots L_{k}(1 \leq k<$ $n)$. Then $G \mid L_{\mathbb{C}}$ is virtually solvable.

(3) $G \mid L_{\mathbb{C}}$ is virtually solvable if and only if there exists a finite-index subgroup $G_{1}$ of $G$ such that $N\left(G_{1}\right) \triangleleft G_{1}$ and $G_{1} / N\left(G_{1}\right) \cong \mathbb{Z}^{\oplus r}$ for some $r \leq n-1$.

We need the following lemmas for the proof of theorems.

Lemma 2.4. Let $G$ be a group, and $H \triangleleft G$ a finite normal subgroup. Then we have:

(1) Suppose that for some $r \geq 1$ and $g_{i} \in G$ we have:

$$
G / H=\left\langle\bar{g}_{1}\right\rangle \times \cdots \times\left\langle\bar{g}_{r}\right\rangle \cong \mathbb{Z}^{\oplus r} .
$$

Then there is an integer $s>0$ such that the subgroup $G_{1}:=\left\langle g_{1}^{s}, \ldots, g_{r}^{s}\right\rangle$ satisfies

$$
G_{1}=\left\langle g_{1}^{s}\right\rangle \times \cdots \times\left\langle g_{r}^{s}\right\rangle \cong \mathbb{Z}^{\oplus r}
$$

and is of finite-index in $G$; further, the quotient map $\gamma: G \rightarrow G / H$ restricts to an isomorphism $\gamma \mid G_{1}: G_{1} \rightarrow \gamma\left(G_{1}\right)$ onto a finite-index subgroup of $G / H$. 
(2) A group is almost abelian of finite rank $r$ if and only if it is virtually abelian of rank $r$.

Proof. (2) follows from (1). For (1), we only need to find $s>0$ such that $g_{i}^{s}$ commutes with $g_{j}^{s}$ for all $i, j$. Since $G / H$ is abelian, the commutator subgroup $[G, G] \leq H$. Thus the commutators $\left[g_{1}^{t}, g_{2}\right](t>0)$ all belong to $H$. The finiteness of $H$ implies that $\left[g_{1}^{t_{1}}, g_{2}\right]=\left[g_{1}^{t_{2}}, g_{2}\right]$ for some $t_{2}>t_{1}$, which implies that $g_{1}^{s_{12}}$ commutes with $g_{2}$, where $s_{12}:=t_{2}-t_{1}$. Similarly, we can find an integer $s_{1 j}>0$ such that $g_{1}^{s_{1 j}}$ commutes with $g_{j}$. Set $s_{1}:=s_{12} \times \cdots \times s_{1 r}$. Then $g_{1}^{s_{1}}$ commutes with every $g_{j}$. Similarly, for each $i$, we can find an integer $s_{i}>0$ such that $g_{i}^{s_{i}}$ commutes with $g_{j}$ for all $j$. Now $s:=s_{1} \times \cdots \times s_{r}$ will do the job. This proves the lemma.

Lemma 2.5. Let $X$ be a compact complex Kähler manifold (resp. normal projective variety), and $G$ a subgroup of $\operatorname{Aut}(X)$. Then, replacing $G$ by a suitable finite-index subgroup, the following are true (cf. 2.1 for notation $\left.L_{\mathbb{C}}\right)$.

(1) There is a normal subgroup $U \triangleleft G$ such that $U \mid L_{\mathbb{C}}$ is unipotent and $G / U$ is embedded as a Zariski-dense subgroup in a reductive complex linear algebraic group.

(2) There is a normal subgroup $R \triangleleft G$ such that $R \mid L_{\mathbb{C}}$ is solvable and $G / R$ is embedded as a Zariski-dense subgroup in a semi-simple complex linear algebraic group $H$.

Proof. Let $\bar{G}$ be the Zariski-closure of $G \mid L_{\mathbb{C}} \subseteq \mathrm{GL}\left(L_{\mathbb{C}}\right)$, and $\iota$ the composite: $G \rightarrow$ $G \mid L_{\mathbb{C}} \rightarrow \bar{G}$. Replacing $G$ by the intersection of $G$ and the $\iota$-inverse of the identity connected component of $\bar{G}$, we may assume that $\bar{G}$ is connected. Let $U(\bar{G})(\operatorname{resp} . R(\bar{G}))$ be the unipotent radical (resp. the radical) of $\bar{G}$. Let $U \leq G$ (resp. $R \leq G$ ) be the $\iota$-inverse of $U(\bar{G})$ (resp. $R(\bar{G})$ ). Then the embeddings $G / U \rightarrow \bar{G} / U(\bar{G})$ and $G / R \rightarrow$ $\bar{G} / R(\bar{G})$, and $U$ and $R$ here meet the requirements of the lemma.

Lemma 2.6. We use the notation $L_{\mathbb{C}}$ of 2.1. A group $G \leq \operatorname{Aut}(X)$ has finite restriction $G \mid L_{\mathbb{C}}$ if and only if the index $\left|G: G \cap \operatorname{Aut}_{0}(X)\right|$ is finite.

Proof. Consider the exact sequence

$$
1 \rightarrow K \rightarrow G \rightarrow G \mid L_{\mathbb{C}} \rightarrow 1
$$

For an ample divisor or Kähler class $\omega$ of $X$, our $K$ is a subgroup of $\operatorname{Aut}_{\omega}(X):=\{g \in$ $\left.\operatorname{Aut}(X) \mid g^{*} \omega=\omega\right\}$, where the latter contains $\operatorname{Aut}_{0}(X)$ as a group of finite-index (cf. [13, Proposition 2.2]). Now the last group below is a finite group

$$
K /\left(K \cap \operatorname{Aut}_{0}(X)\right) \cong\left(K \operatorname{Aut}_{0}(X)\right) / \operatorname{Aut}_{0}(X) \leq \operatorname{Aut}_{\omega}(X) / \operatorname{Aut}_{0}(X) .
$$

The lemma follows since the connected group $\operatorname{Aut}_{0}(X)$ acts trivially on the lattice $L$ (and hence on $L_{\mathbb{C}}$ ) so that $G \cap \operatorname{Aut}_{0}(X)=K \cap \operatorname{Aut}_{0}(X)$. 
A more precise version of 2.7 below was proved in [6, 6.1] for finitely generated groups.

Lemma 2.7. Let $G$ be a group of automorphisms of a compact Kähler manifold $X$. Consider an exact sequence of groups:

$$
1 \rightarrow N \rightarrow G \rightarrow Q \rightarrow 1
$$

Suppose $N$ is contained in the union of finitely many connected components of $\operatorname{Aut}(X)$. Suppose both $N$ and $Q$ are virtually solvable. Then $G$ is also virtually solvable.

Proof. Let $\bar{N} \subseteq \operatorname{Aut}(X)$ be the Zariski-closure of $N$. Replacing $G$ by a suitable finiteindex subgroup, we may assume that $Q$ is solvable. Since $\bar{N} \cap G \triangleleft G$, we have $(\bar{N})_{0} \cap G \triangleleft G$ for the identity connected component $(\bar{N})_{0}$ of $\bar{N}$. Hence $M:=(\bar{N})_{0} \cap N \triangleleft G$. Now

$$
N / M \cong\left(N(\bar{N})_{0}\right) /(\bar{N})_{0} \leq \bar{N} /(\bar{N})_{0}
$$

where the latter is a finite group. We have an exact sequence

$$
1 \rightarrow N / M \rightarrow G / M \rightarrow G / N=Q \rightarrow 1 .
$$

Replacing $G / M$ by a finite-index subgroup we may assume that the conjugate action of $G / M$ (and hence of $N / M$ ) on the finite group $N / M$ is trivial. Thus $N / M$ is abelian and hence $G / M$ is solvable. Since $N$ is virtually solvable so is $\bar{N}$. Hence $(\bar{N})_{0}$ is solvable. Thus $M$ is solvable. Therefore, $G$ is solvable.

Lemma 2.8. Let $X$ be a compact complex Kähler manifold (resp. normal projective variety), and $G \leq \operatorname{Aut}(X)$ a subgroup. Assume the following two conditions:

(1) $H \triangleleft G$; and $H$ has a finite-index subgroup $H_{1}$ such that the null set $N\left(H_{1}\right)$ is a (normal) subgroup of $H_{1}$ and $H_{1} / N\left(H_{1}\right)=\langle\bar{h}\rangle \cong \mathbb{Z}$ for some $h \in H_{1}$.

(2) Suppose that there is a common nef eigenvector $L_{1}$ of $H_{1}$, and further that $h^{*} L_{1}=$ $d_{1}(h) L_{1}$, i.e., $L_{1}$ equals some $L_{h}$ up to scalar; suppose also that for every $s \neq 0$ and every nef $M$ so that $\left(h^{s}\right)^{*} M=\lambda M$ with $\lambda \neq 1$, we have $M$ parallel to either one of $L_{h^{ \pm 1}}$ (which are two fixed nef eigenvectors).

Then the stabilizer subgroup $\operatorname{Stab}_{L_{h}}(G):=\left\{g \in G \mid g^{*} L_{h}\right.$ is parallel to $\left.L_{h}\right\}$ has index $\leq 2$ in $G$.

Proof. We begin with:

Claim 2.9. For every $g \in G$, the class $g^{*} L_{h}$ is parallel to one of $L_{h^{ \pm 1}}$.

We prove the claim. Take any element $g$ of $G$. Since $H \triangleleft G$, we have $g h g^{-1} \in H \backslash N(H)$. Hence $g h^{a} g^{-1}$ is in $H_{1}$ with $a=\left|H: H_{1}\right|$, so it equals $h^{b} n$ for some $b \neq 0$ and $n \in N\left(H_{1}\right)$. Since the element $n$ of $N\left(H_{1}\right)$ fixes $L_{1}=L_{h}$ (cf. 2.1) ,

$$
\left(g h^{a} g^{-1}\right)^{*} L_{h}=d_{1}(h)^{b} L_{h}, \quad\left(h^{a}\right)^{*}\left(g^{*} L_{h}\right)=d_{1}(h)^{b}\left(g^{*} L_{h}\right) .
$$


Now the condition (2) applied to $h^{a}$ and $\lambda:=d_{1}(h)^{b} \neq 1$, implies the claim.

Return to the proof of Lemma 2.8. Suppose there is some $g_{1} \in G \backslash \operatorname{Stab}_{G}\left(L_{h}\right)$. Take any $g \in G \backslash \operatorname{Stab}_{G}\left(L_{h}\right)$. By Claim 2.9, we have (with equalities all up to scalars) $g^{*} L_{h}=L_{h^{-1}}=$ $g_{1}^{*} L_{h},\left(g g_{1}^{-1}\right)^{*} L_{h}=\left(g_{1}^{-1}\right)^{*} g^{*} L_{h}=L_{h}$. Hence $g g_{1}^{-1} \in \operatorname{Stab}_{G}\left(L_{h}\right)$ and $g=\left(g g_{1}^{-1}\right) g_{1} \in$ $\operatorname{Stab}_{G}\left(L_{h}\right) g_{1}$. So $G=\operatorname{Stab}_{G}\left(L_{h}\right) \cup \operatorname{Stab}_{G}\left(L_{h}\right) g_{1}$. The lemma follows.

\subsection{Proof of Theorem 2.2}

Assertion (2) follows from Assertion (1), so we only need to prove Theorem 2.2(1).

We follow the proof of Oguiso [16, Prop 2.2]. Since $G$ is of null entropy, the subset

$$
U:=\left\{g \in G ; g \mid L_{\mathbb{C}} \text { is unipotent }\right\}
$$

is a normal subgroup of $G$. If $G \mid L_{\mathbb{C}}$ is not virtually solvable, then by the classical Tits alternative theorem [19, Theorem 1], there are $g_{i} \in G$ such that $\left\langle g_{1}, g_{2}\right\rangle \mid L_{\mathbb{C}}=$ $\left(\left\langle g_{1}\right\rangle \mid L_{\mathbb{C}}\right) *\left(\left\langle g_{2}\right\rangle \mid L_{\mathbb{C}}\right)=\mathbb{Z} * \mathbb{Z}$. As observed in [16], $g_{i}^{s} \in U$ for some $s \geq 1$, and hence $\mathbb{Z} * \mathbb{Z}=\left\langle g_{1}^{s}, g_{2}^{s}\right\rangle\left|L_{\mathbb{C}} \leq U\right| L_{\mathbb{C}}$ which is unipotent (and hence solvable). This is absurd.

Thus, $G \mid L_{\mathbb{C}}$ is virtually solvable. Replacing $G$ by a suitable finite-index subgroup, we may assume that $G \mid L_{\mathbb{C}}$ is solvable and its closure $\bar{G}$ in $\operatorname{GL}\left(L_{\mathbb{C}}\right)$ is connected (and solvable). Write $\bar{G}=\bar{U} \rtimes \bar{T}$ where $\bar{U}$ is the unipotent radical and $\bar{T}$ a maximal torus in $\bar{G}$. As observed in [16], the image of $G$ via the quotient map $\bar{G} \rightarrow \bar{T}$ is a torsion group in $\operatorname{GL}\left(L_{\mathbb{C}}\right)$ with bounded exponent and hence a finite group by Burnside's theorem. Thus the index $|G: U|<\infty$.

To finish the proof of Assertion (1), we may assume that $G=U$ and it suffices to show that $G \mid L$ is generated by $\ell(\ell-1) / 2$ elements where $\ell=\operatorname{rank} L$. Regarding $\bar{G}$ as a subgroup of upper triangular matrices, there is a standard normal series

$$
1 \triangleleft U_{1} \triangleleft U_{2} \triangleleft \cdots \triangleleft U_{\ell(\ell-1) / 2}=\bar{G}
$$

such that the factor groups are all 1-dimensional. Restricting the series to $G \mid L$, we get a normal series of discrete groups whose factor groups are cyclic groups. Thus $G \mid L$ is generated by $\ell(\ell-1) / 2$ elements. This proves Theorem 2.2 .

\subsection{Proof of Theorem 2.3}

(1) was proved in [23, Theorem 1.2]. For (2), suppose that $G$ is polarized by a quasi-nef sequence $L_{1} \cdots L_{k}(1 \leq k<n)$ so that $g^{*}\left(L_{1} \cdots L_{k}\right)=\chi_{1}(g) \cdots \chi_{k}(g) L_{1} \cdots L_{k}$. As in the proof of [23, Theorem 1.2], the homomorphism

$$
\varphi: G \rightarrow(\mathbb{R},+), \quad g \mapsto\left(\log \chi_{1}(g), \ldots, \log \chi_{n-1}(g)\right)
$$

has $\operatorname{Ker}(G)=N(G)$, and $\varphi(G)=\mathbb{Z}^{\oplus r}$ a lattice in $\mathbb{R}^{n-1}$. By Theorem 2.2, $N(G) \mid L_{\mathbb{C}}$ is virtually solvable, so is $G \mid L_{\mathbb{C}}$, since $G / N(G)$ is abelian and by Lemma 2.7 . 
For (3), the "if part" follows from Theorem 2.2 and Lemma 2.7. The "only if" part is by [23, Theorem 1.2, Remark 1.3]. This proves Theorem 2.3 .

\subsection{Proof of Theorem 1.5}

We may assume that Assertion (1) is not satisfied. Replacing $G$ by a suitable finiteindex subgroup and by [19, Thm 1], we may assume that $G \mid L_{\mathbb{C}}$ is solvable and its closure $\bar{G}$ in $\operatorname{GL}\left(L_{\mathbb{C}}\right)$ is connected (and solvable). Let $K=\operatorname{Ker}\left(G \rightarrow G \mid L_{\mathbb{C}}\right.$ ) be as in Lemma 2.6.

Suppose that $K$ is virtually solvable. Then so is $G$ by Lemma 2.7. Thus Theorem 1.5)(3) occurs, by [23, Theorem 1.2, Remark 1.3].

Suppose that $K$ is not virtually solvable. Consider the exact sequence

$$
1 \rightarrow L_{A} \rightarrow \operatorname{Aut}_{0}(X) \rightarrow T \rightarrow 1
$$

where $L_{A}$ is the linear part of $\operatorname{Aut}_{0}(X)$ and $T$ a compact complex torus (cf. [13, Theorem 3.12]). This induces the exact sequence (with $Q$ abelian):

$$
1 \rightarrow K \cap L_{A} \rightarrow K \cap \operatorname{Aut}_{0}(X) \rightarrow Q \rightarrow 1 .
$$

We may assume that Theorem 1.5)(2) does not occur. So $K \cap L_{A}$ is virtually solvable by Tits alternative. Thus so is $K \cap \operatorname{Aut}_{0}(X)$ by the exact sequence above and Lemma 2.7. Now $K /\left(K \cap \operatorname{Aut}_{0}(X)\right)$ is a finite group by Lemma 2.6. Hence $K$ is also virtually solvable, contradicting our extra assumption.

For the final assertion, replacing $G$ by a suitable finite-index subgroup, we may assume that $G \mid L_{\mathbb{C}}$ is solvable and has connected Zariski-closure in $\operatorname{GL}\left(L_{\mathbb{C}}\right)$. Then $G / N(G) \cong$ $\mathbb{Z}^{\oplus r}$ by [23, Theorem 1.2]. This and Theorem 2.2 for $N(G)$ imply the assertion.

\section{Strong PRIMitivity FOR ThreEFolds}

We prove Theorem 1.2. Replacing $G_{0}$ by the identity connected component of its Zariski-closure in $\operatorname{Aut}_{0}(X)$ we may further assume that $G_{0}=G \cap \operatorname{Aut}_{0}(X)$ is connected, positive-dimensional and closed in $\operatorname{Aut}_{0}(X)$. Because $G$ acts naturally on the quotient of $X$ modulo $G_{0}$ and because of our assumption, we may assume that one orbit of $G_{0}$ is a Zariski-dense open subset of $X$, i.e., $X$ is almost homogeneous (cf. [23, Lemma 2.14]).

Claim 3.1. Suppose the irregularity $q(X)=h^{1}\left(X, \mathcal{O}_{X}\right)>0$. Then Theorem 1.2 is true.

We prove Claim 3.1. By the proof of [23, Lemma 2.13], the albanese map $a: X \rightarrow A:=$ $\operatorname{Alb}(X)$ is surjective, birational and necessarily $\operatorname{Aut}(X)$-equivariant. Our $G_{0}$ induces an action on $A$ and we denote it by $G_{0} \mid A$. Since $G_{0} \mid A$ also has a Zariski-dense open orbit in $A$, we have $G_{0} \mid A=\operatorname{Aut}_{0}(X)(\cong A)$. Let $B \subset A$ be the locus over which $a$ is not an isomorphism. Note that $B$ and $a^{-1}(B)$ are $G_{0}$-stable. Since $G_{0} \mid A=\operatorname{Aut}_{0}(X)$, we have $B=\emptyset$. Claim 3.1 is proved. 
We continue the proof of Theorem [1.2. By Claim [3.1, we may assume that $q(X)=0$. Thus $G_{0} \leq \operatorname{Aut}_{0}(X)$ is a linear algebraic group and has a Zariski-dense open orbit in $X$. In particular, $X$ is ruled and unirational, because linear algebraic groups are rational varieties by a classical result of Chevalley.

In the rest of the proof, we shall derive a contradiction. Let $U \subseteq X$ be the open dense $G_{0}$-orbit and $F:=X \backslash U$. Then $F$ consists of finitely many prime divisors and some subvarieties of codimension $\geq 2$. Since $G_{0} \triangleleft G$, we may assume that both $U$ and all irreducible components of $F$ are $G$-stable, after replacing $G$ by a suitable finite-index subgroup. $X$ has only finitely many $G_{0}$-periodic prime divisors, all of which are contained in $F$ and $G$-stable.

By the minimal model program (MMP) in dimension three (cf. [12, §3.31, §3.46]), the end product of a uniruled variety (like our $X$ here) is an extremal Fano contraction $f: X_{m} \rightarrow Y$ with a general fibre $X_{m, y}$, i.e., by definition, the restriction $-K_{X_{m}} \mid X_{m, y}$ of the canonical divisor $-K_{X_{m}}$ is ample and the Picard numbers satisfy $\rho\left(X_{m}\right)=1+\rho(Y)$.

Claim 3.2. (1) Every $G_{0}$-periodic subvariety of $X$ is actually $G_{0}$-stable.

(2) There are a composite $X=X_{0} \rightarrow X_{1} \cdots \rightarrow X_{m}$ of birational extremal contractions and an extremal Fano contraction $X_{m} \rightarrow Y$ with $\operatorname{dim} Y<\operatorname{dim} X$. The induced birational action of $G_{0}$ on each $X_{i}$ is biregular. $G_{0} \mid X_{m}$ descends to an action on $Y$ so that $X_{m} \rightarrow Y$ is $G_{0}$-equivariant.

(3) In (2), for every finite-index subgroup $G_{1}$ of $G$, there is at least one $i \in\{1, \ldots, m\}$ such that the induced action of $G_{1}$ on $X_{i}$ is not biregular.

(4) In (2), let $s \leq m$ be the largest integer such that $X_{i} \rightarrow X_{i+1}$ is divisorial for every $i \in\{0,1, \ldots, s-1\}$. Then, replacing $G$ by a suitable finite-index subgroup, the induced birational action of $G$ on each $X_{i}(i<s)$ is biregular and hence each map $X_{i-1} \rightarrow X_{i}$ is G-equivariant. In particular, $s<m$.

Remark 3.3. By the choice of $s$ in (4), $X_{s} \rightarrow X_{s+1}$ is a flip with a flipping contraction $X_{s} \rightarrow Y_{s}$ and with $X_{s+1}=\operatorname{Proj}_{Y_{s}}\left(\oplus_{m \geq 0} \mathcal{O}_{Y_{s}}\left(m K_{Y_{s}}\right)\right.$ ) (cf. [12, Cor 6.4 or Thm 3.52]).

We now prove Claim 3.2. (1) is true because $G_{0}$ is a connected group. For the first part of $(2)$, see [12, $§ 3.31, \S 3.46]$ when $\operatorname{dim} X=3$ and [2, Corollary 1.3.2] when $\operatorname{dim} X$ is arbitrary. The second part of $(2)$ is true because $G_{0}$ acts trivially on $H^{i}(X, \mathbb{Z})$, and also on $\mathrm{NS}_{\mathbb{C}}(X)$ and the extremal rays of $\overline{\mathrm{NE}}(X)$ (cf. [25, Lemmas 2.12 and 3.6]).

For (4), suppose that $X=X_{0} \rightarrow X_{1}$ is a divisorial contraction of an extremal ray $R:=\mathbb{R}_{>0}[\ell]$ with an exceptional divisor $D_{0}$. Since $G_{0}$ acts trivially on the extremal rays of $\overline{\mathrm{NE}}(X)$, this $D_{0}$ is $G_{0}$-stable. So $D_{0}$ is contained in $F$ and is $G$-stable.

Since the natural map $G / G_{0} \rightarrow \operatorname{Aut}\left(H^{2}(X, \mathbb{Z})\right.$ ) has finite kernel (cf. [13, Proposition $2.2]$ ) and by the assumption, $G / G_{0}$ is almost abelian of finite rank $r>0$. By Lemma 2.4 
and replacing $G$ by a finite-index subgroup, there is some $H_{0} \triangleleft G$ such that $H_{0}$ contains $G_{0}$ as a subgroup of finite index and $G / H_{0}=\left\langle\bar{g}_{1}\right\rangle \oplus \cdots \oplus\left\langle\bar{g}_{r}\right\rangle \cong \mathbb{Z}^{\oplus r}$ for some $g_{i} \in G$.

In [25, Lemma 3.7], it is proved that a positive power of $g_{i}$ preserves the extremal ray $R$ and hence descends to a biregular automorphism of $X_{1}$. Thus $X \rightarrow X_{1}$ is $G$-equivariant after $G$ is replaced by a finite-index subgroup. Indeed, we may assume that $g_{i}(R)=R$ so that $\{g(R) \mid g \in G\}$ consists of no more than $\left|H_{0}: G_{0}\right|$ extremal rays so that a finite-index subgroup of $G$ fixes $R$. This and the second sentence of the next paragraph are the places where we need $G / G_{0}$ to be almost abelian.

For (3), suppose the contrary that $G$ (replaced by a finite-index subgroup) acts biregularly on all $X_{i}$. Then, as in the proof of (4) above, by [25, Theorem 2.13, or Appendix], we may assume that $G$ (replaced by its finite-index subgroup) fixes the extremal ray giving rise to the extremal Fano contraction $X_{m} \rightarrow Y$, and hence $X_{m} \rightarrow Y$ is $G$-equivariant. By the strong primitivity assumption, we have $\operatorname{dim} Y=0$, so the Picard number $\rho\left(X_{m}\right)=1$ and $-K_{X_{m}}$ is ample. Since $G$ fixes the ample class of $-K_{X_{m}}$, it is a finite extension of $G_{0}$ (cf. [13, Proposition 2.2]). This contradicts the assumption. Claim 3.2 is proved.

Claim 3.4. It is impossible that $\mathrm{NS}_{\mathbb{C}}\left(X_{i}\right)$ with $0 \leq i \leq m$ is spanned by $-K_{X_{i}}$ and $G_{0}$-periodic divisors, or that $\mathrm{NS}_{\mathbb{C}}(Y)$ is spanned by $G_{0}$-periodic divisors.

Indeed, note that $\mathrm{NS}_{\mathbb{C}}\left(X_{m}\right)$ is spanned by $-K_{X_{m}}$ (which is ample over $Y$ ) and the pullback of $\mathrm{NS}_{\mathbb{C}}(Y)$, and $\mathrm{NS}_{\mathbb{C}}(X)$ is spanned by the pullback of $\mathrm{NS}_{\mathbb{C}}\left(X_{i}\right)$ and (necessarily $G_{0}$-stable) exceptional divisors of $X \rightarrow X_{i}$. Thus we only need to rule out the possibility that $\mathrm{NS}_{\mathbb{C}}(X)$ is spanned by $-K_{X}$, and $G_{0}$-stable divisors $D_{i}$ all of which are necessarily contained in $F$ and hence $G$-stable.

Write an ample divisor $M$ on $X$ as a combination of $-K_{X}$ and $D_{i}$ 's. Then $G \leq$ $\operatorname{Aut}_{[M]}(X)$, so $\left|G / G_{0}\right|<\infty$ as in the proof of Lemma 2.6, contradicting the assumption.

Claim 3.5. $X_{m}$ and hence $Y$ contain a $G_{0}$-fixed point (here we use that $\operatorname{dim} X=3$ ).

Indeed, note that a smooth threefold has no flip and a flip preserves the singularity type of a threefold. By Claim [3.2, for some $m-1 \geq t \geq s, X_{t}-\rightarrow X_{t+1}$ is a flip and $X_{t+1} \rightarrow \cdots \rightarrow X_{m}$ is the composite of extremal divisorial contractions. So the non-empty finite set Sing $X_{t+1}$ (cf. [12, Corollary 5.18]) and its image on $X_{m}$ are fixed by $G_{0}$.

Claim 3.6. It is impossible that $\operatorname{dim} Y \leq 1$.

Indeed, if $\operatorname{dim} Y=0$, then $\mathrm{NS}_{\mathbb{C}}\left(X_{m}\right)$ is of rank one and spanned by $-K_{X_{m}}$ (which is ample over $Y$ ). This contradicts Claim 3.4. If $\operatorname{dim} Y=1$ then the rank two space $\mathrm{NS}_{\mathbb{C}}\left(X_{m}\right)$ is spanned by $-K_{X_{m}}$ (which is ample over $Y$ ) and the fibre over a $G_{0}$-fixed point $y_{0}$ (cf. Claim 3.5). This contradicts Claim 3.4. 
We continue the proof of Theorem 1.2. Take an extremal ray on $X_{s}$ generated by a rational curve $\ell$ and let $X_{s} \rightarrow X_{s+1}$ be the flip (cf. Claim 3.2 for $s$ ). Note that $G_{0}$ stabilizes all irreducible components $E_{i}$ of the exceptional locus of the flipping contraction $X_{s} \rightarrow Y_{s}$ and $G$ (replaced by a finite-index subgroup) stabilizes all irreducible components $D_{i j}$ of the Zariski closure of $\cup_{g \in G} g\left(E_{i}\right)$, because $G_{0} \triangleleft G$. These $D_{i j}$ are unions of 'small' $G_{0}$-orbits and hence are contained in the image of the algebraic subset $F \subset X$.

If $\operatorname{dim} D_{i j}=\operatorname{dim} E_{i}=1$, then $G$ preserves the extremal ray $\mathbb{R}_{\geq_{0}}[\ell] \subseteq \overline{\mathrm{NE}}\left(X_{s}\right)$ and we can descend $G$ to a biregular action on $X_{s+1}$ (cf. [25, Lemma 3.6]). Now apply MMP on $X_{s+1}$ and continue the process.

Assume that $\operatorname{dim} D_{i j}=2>\operatorname{dim} E_{i}=1$. If $G_{0}$ acts trivially on some $g_{0} E_{i}$ in the set $\left\{g E_{i} \mid g \in G\right\}$, then $G_{0}=g G_{0} g^{-1}$ acts trivially on $g g_{0} E_{i}$, i.e., on all $g^{\prime} E_{i}\left(g^{\prime} \in G\right)$. Hence $G_{0} \mid D_{i j}=$ id. This contradicts Claim 3.7 below.

Suppose that $G_{0}$ acts non-trivially on some $g_{0} E_{i}$ and hence on all $g E_{i}(g \in G)$. Then these extremal curves $g E_{i}$ are fibres of the quotient map $D_{i j} \rightarrow D_{i j} / G_{0}=: B$ over a curve $B$, hence homologous to each other. So they give rise to one and the same class in the extremal ray $\mathbb{R}_{\geq 0}[\ell] \subseteq \overline{\mathrm{NE}}\left(X_{s}\right)$. Thus $G$ preserves this extremal ray and we can descend $G$ to a biregular action on $X_{s+1}$ (cf. [25, Lemma 3.6]). Now apply MMP on $X_{s+1}$ and continue the process. Therefore, we can continue the $G$-equivariant MMP and reach an extremal Fano fibration $X_{m} \rightarrow Y$ which is a contradiction (cf. Claim 3.2).

To complete the proof of Theorem 1.2, we still need to prove:

Claim 3.7. It is impossible that $\operatorname{dim} Y=2, \operatorname{dim} D_{i j}=2$ and $G_{0} \mid D_{i j}=\mathrm{id}$.

We now prove Claim 3.7. $X_{m} \rightarrow Y$ is known as an extremal conic fibration. We may take a $G_{0}$-equivariant blowup $\hat{X} \rightarrow X_{s}$ to resolve indeterminacy of the composite $\pi_{s}: X_{s} \rightarrow X_{m} \rightarrow Y$ so that the induced map $\hat{\pi}: \hat{X} \rightarrow Y$ is holomorphic and $G_{0^{-}}$ equivariant. By [15, Theorem 4.8], there exist blowups $\sigma_{x}: X^{\prime} \rightarrow \hat{X}$ and $\sigma_{y}: Y^{\prime} \rightarrow Y$ with $X^{\prime}$ and $Y^{\prime}$ smooth, and extremal conic fibration $\pi^{\prime}: X^{\prime} \rightarrow Y^{\prime}$ such that $\hat{\pi} \circ \sigma_{x}=\sigma_{y} \circ \pi^{\prime}$. We may also assume that the four maps above are $G_{0}$-equivariant by taking extra blowups so that they are equivariant (noting that $G_{0}$ stabilizes extremal rays).

We will reach a contradiction to Claim 3.4. To do so, we consider both $Y$ and $Y^{\prime}$.

Indeed, if $K_{Y^{\prime}}^{2} \leq 7$, then $\mathrm{NS}_{\mathbb{C}}\left(Y^{\prime}\right)$ (and hence $\mathrm{NS}_{\mathbb{C}}(Y)$ ) are spanned by $G_{0}$-stable curves (i.e., the negative curves on $Y^{\prime}$ ). This contradicts Claim 3.4. Therefore, we may assume that $K_{Y^{\prime}}^{2}=9$ or 8 , and $Y^{\prime}=\mathbb{P}^{2}$ or a Hirzebruch surface $F_{d}$ of degree $d \geq 0$.

If $Y^{\prime}=\mathbb{P}^{2}$ or $Y^{\prime}=\mathbb{P}^{1} \times \mathbb{P}^{1}$, then $Y^{\prime}$ has no negative curve to contract, so $Y^{\prime}=Y$.

If $Y=F_{d}$ then $G_{0}$ stabilizes a fibre passing through a fixed point of $y_{0}$ of $G_{0} \mid Y$ (cf. Claim 3.5), and the zero-section through $y_{0}$ (resp. the unique $(-d)$-curve) when $d=0$ (resp. $d \geq 1$ ). This contradicts Claim 3.4. 
Therefore, we may assume that either $Y=Y^{\prime}=\mathbb{P}^{2}$, or $F_{d}=Y^{\prime} \rightarrow Y$ (with $d \geq 1$ ) is the contraction of the unique $(-d)$-curve. Thus the Picard number $\rho\left(X_{m}\right)=1+\rho(Y)=2$.

Let $D_{i j}^{\prime} \subset X^{\prime}$ be the proper transform of $D_{i j} \subset X_{s}$. Then $G_{0}$ acts trivially on $D_{i j}^{\prime}$ because so does $G_{0}$ on $D_{i j}$. Since every fibre of $\pi^{\prime}: X^{\prime} \rightarrow Y^{\prime}$ is 1-dimensional, the image $C_{i j} \subseteq Y^{\prime}$ of $D_{i j}^{\prime}$ is the whole $Y^{\prime}$ or a curve, and $G_{0} \mid C_{i j}=$ id. Since $G_{0} \mid Y=$ id would contradict Claim 3.4, we may assume that $C_{i j}$ is a curve in $Y^{\prime}$. If $Y^{\prime}=Y=\mathbb{P}^{2}$ (resp. $Y^{\prime}=F_{d} \rightarrow Y$ is the contraction of the $(-d)$-curve), then $G_{0} \mid Y$ stabilizes $C_{i j}$ (resp. the image of $C_{i j}$ or every generating line). This contradicts Claim 3.4. Claim 3.7 is proved.

Corollary 3.8. Let $X$ be a 3-dimensional normal projective variety and $G \leq \operatorname{Aut}(X)$ a subgroup of null entropy such that $G_{0}:=G \cap \operatorname{Aut}_{0}(X)$ is infinite and the quotient $G / G_{0}$ is an almost abelian group of positive rank. Then $(X, G)$ is not strongly primitive.

Proof. Taking a $G$-equivariant resolution, we may assume that $X$ is smooth. With our assumption and the proof of Theorem 1.2, we may assume that $X$ is a complex torus, and $G_{0}$ is connected, is closed and has a Zariski-dense open orbit in $X$. Thus $G_{0}=\operatorname{Aut}_{0}(X)$. By Lemma 2.4 and replacing $G$ by a suitable finite-index subgroup, we may assume that $G / G_{0}$ is abelian and equals $\left\langle\bar{g}_{1}, \ldots, \bar{g}_{r}\right\rangle$ for $g_{i} \in G$, where the order $o\left(\bar{g}_{i}\right)=\infty$; moreover, $g_{i}$ has unipotent representation matrix on $H^{0}\left(X, \Omega_{X}^{1}\right)$ using Kronecker's theorem as in [24, Lemma 2.14]. Write $g_{i}=T_{t_{i}} \circ h_{i}$ where $T_{t_{i}}$ is the translation by $t_{i}$ and $h_{i}$ is a group automorphism. As in [24, Lemma 2.15], the identity connected component $B$ of the fixed locus $X^{h_{1}}$ (pointwise) has dimension equal to that of $\operatorname{Ker}\left(h_{1}^{*}-\mathrm{id}\right) \subset H^{0}\left(X, \Omega_{X}^{1}\right)$ and is hence between 1 and $\operatorname{dim} X-1$. Note that $h_{1} h_{j}=h_{j} h_{1}$ holds modulo $\operatorname{Aut}_{0}(X)$ and hence holds in $\operatorname{Aut}(X)$ since both sides fix the origin. Thus $h_{j}(B)$ is contained in $X^{h_{1}}$ and hence equals $B$ since it contains the origin. Now $g_{j}(x+B)=g_{j}(x)+B$. So $g_{j}$ permutes cosets of the quotient torus $X / B$; the same is true for elements of $\operatorname{Aut}_{0}(X)$. Thus, the quotient map $X \rightarrow X / B$ is $G$-equivariant. This proves Corollary 3.8 .

\subsection{Proof of Corollary 1.3}

Taking a $G$-equivariant resolution, we may assume that $X$ is smooth. If $q(X)>0$, then, by Claim 3.1, we may assume that $X$ is a complex torus so that $\operatorname{Aut}_{0}(X) \neq\left\{\operatorname{id}_{X}\right\}$. Thus, we may always assume that $\operatorname{Aut}_{0}(X) \neq\left\{\operatorname{id}_{X}\right\}$.

Replacing $G$ by $G$. $\operatorname{Aut}_{0}(X)$ we may assume that $G \geq G_{0}:=\operatorname{Aut}_{0}(X)$. According to Lemma 2.6, we have $G \mid \mathrm{NS}_{\mathbb{C}}(X)=G / K$ where $\left|K / G_{0}\right|<\infty$. Thus $G / G_{0}$ is also almost abelian of positive rank by assumption. Now Corollary 1.3 follows from Corollary 3.8 .

\section{Minimal threefolds}

Below sufficient conditions for being a quotient of a torus are given. 
Theorem 4.1. Let $X$ be a 3-dimensional minimal projective variety. Assume that one of the following two properties is satisfied:

(1) The first Chern class $c_{1}(X)=0$. The second Chern class $c_{2}(X)$ (as a linear form on $\mathrm{NS}_{\mathbb{C}}(X)$ as in [18, p. 265]) has zero intersection with a nef and big $\mathbb{R}$-divisor.

(2) There is a subgroup $G \leq \operatorname{Aut}(X)$ such that the null set $N(G)$ is a subgroup of $G$ and $G / N(G) \cong \mathbb{Z}^{\oplus 2}$.

Set $B:=\operatorname{Aut}(X)$. Then there is a B-equivariant birational surjective morphism $X \rightarrow X^{\prime}$ such that $X^{\prime} \cong T / F$ for a finite group $F$ acting freely outside a finite set of an abelian variety $T$ of dimension three. Further, the action of $B$ on $X^{\prime}$ lifts to an action of a group $\widetilde{B}$ on $T$ such that $\widetilde{B} / F \cong B$.

Proof. Assume the condition (1) in Theorem 4.1. Let

$$
D:=\operatorname{Nef}(X) \cap c_{2}(X)^{\perp}=\left\{M \in \operatorname{Nef}(X) \mid M \cdot c_{2}(X)=0\right\}
$$

be a closed subcone of the nef cone $\operatorname{Nef}(X)$ of $X$. Let

$$
C:=\overline{\mathrm{NE}}(X) \cap D^{\perp}=\left\{[\ell] \in \overline{\mathrm{NE}}(X) \mid \ell \cdot D_{0}=0 \text { for all } D_{0} \in D\right\}
$$

be a closed subcone of the closed cone $\overline{\mathrm{NE}}(X)$ of effective curves on $X$. Then $c_{2}(X) \in C$ by definition and using Miyaoka's pseudo-effectivity of $c_{2}$ for any minimal variety $X$ of dimension $n$ : $c_{2}(X) \cdot\left(H_{1} \cdots H_{n-2}\right) \geq 0$ for all nef divisors $H_{i}$ on $X$ (cf. [18, Theorem 4.1, Proposition 1.1]).

By assumption, $D$ contains a nef and big $\mathbb{R}$-divisor. Let $A$ be an interior element of D. As in [2, Theorem 3.9.1], there is a birational contraction

$$
\sigma: X \rightarrow X^{\prime}
$$

such that a curve $\ell \subset X$ is contracted to a point if and only if the class $[\ell]$ is contained in $C$, and such that $A=\sigma^{*} A^{\prime}$ for some ample $\mathbb{R}$-divisor $A^{\prime}$.

By the projection formula and since $A$ is contained in $D$, we have $A^{\prime} \cdot c_{2}\left(X^{\prime}\right)=\sigma^{*} A^{\prime} \cdot c_{2}(X)$ $=A \cdot c_{2}(X)=0$. For any ample $\mathbb{R}$-divisor $P$ on $X^{\prime}$, a small perturbation $A_{\varepsilon}^{\prime}:=A^{\prime}-\varepsilon P$ of the ample divisor $A^{\prime}$ is still ample because the ample cone of $X^{\prime}$ is open. By Miyaoka's pseudo-effectivity of $c_{2}$ for minimal variety, we have

$$
0 \leq \varepsilon P \cdot c_{2}\left(X^{\prime}\right) \leq\left(A_{\varepsilon}^{\prime}+\varepsilon P\right) \cdot c_{2}\left(X^{\prime}\right)=A^{\prime} \cdot c_{2}\left(X^{\prime}\right)=0 .
$$

So P. $c_{2}\left(X^{\prime}\right)=0$. Since $\mathrm{NS}_{\mathbb{C}}\left(X^{\prime}\right)$ is spanned by ample divisors, we obtain then $c_{2}\left(X^{\prime}\right)=0$ as a linear form on $\mathrm{NS}_{\mathbb{C}}\left(X^{\prime}\right)$.

Thus, $c_{1}(X)$ and $c_{2}(X)$ vanish, and by [18, Corollary, p. 266], we have $X^{\prime}=T / F$ where $F$ is a finite group acting on the abelian variety $T$ freely outside a finite set. Since $D$ and hence $C$ are stable under the action of $B:=\operatorname{Aut}(X)$, the contraction 
$\sigma: X \rightarrow X^{\prime}$ is $B$-equivariant. By [1, §3, especially Proposition 3] applied to étale-incodimension-one covers, replacing $T$ by the finite cover corresponding to the maximal lattice in $\pi_{1}\left(X^{\prime} \backslash\right.$ Sing $\left.X^{\prime}\right)$, we can lift the action of $B$ on $X^{\prime}$ to an action of a group $\widetilde{B}$ on $T$ such that $\widetilde{B} / \operatorname{Gal}\left(T / X^{\prime}\right) \cong B$. This proves Theorem 4.1 under condition (1).

Next, assume condition (2) in Theorem 4.1. The maximality of the rank of $G / N(G)$ and [23, Lemma 2.11] imply the Kodaira dimension $\kappa(X)=0$. The abundance theorem for minimal threefolds implies $K_{X} \sim_{\mathbb{Q}} 0$ (cf. [12, 3.13]). Replacing $G$ by a finite-index subgroup, we may assume that $G \mid \mathrm{NS}_{\mathbb{C}}(X)$ is solvable and has connected Zariski-closure in $\mathrm{GL}\left(\mathrm{NS}_{\mathbb{C}}(X)\right.$ ) (cf. Theorem 2.2 or 2.3). By [26, Claim 2.5(1)], $c_{2}(X)$ is perpendicular to a nef and big $\mathbb{R}$-divisor. We are reduced to condition (1). This proves Theorem 4.1.

The next is the key step towards Theorem 1.1.

We recall the notation in the Introduction: For a subgroup $G$ of $\operatorname{Aut}(X)$, let $\bar{G} \subseteq$ $\mathrm{GL}\left(\mathrm{NS}_{\mathbb{C}}(X)\right)$ be the Zariski-closure of $G \mid \mathrm{NS}_{\mathbb{C}}(X)$ and $R(\bar{G})$ its solvable radical, both of which are defined over $\mathbb{Q}$. We have a natural composition of homomorphisms:

$$
\iota: G \rightarrow G \mid \mathrm{NS}_{\mathbb{C}}(X) \rightarrow \bar{G} .
$$

Theorem 4.2. Let $X$ be a 3-dimensional minimal projective variety and $G \leq \operatorname{Aut}(X)$ a subgroup such that $G \mid \mathrm{NS}_{\mathbb{C}}(X)$ is not virtually solvable. Then we have:

(1) Suppose that $R(G):=\iota^{-1}(\iota(G) \cap R(\bar{G}))$ is of null entropy. Then $R(G) \mid \operatorname{NS}_{\mathbb{C}}(X)$ is virtually unipotent and hence of null entropy. Replacing $G$ by a suitable finiteindex subgroup, $G / R(G)$ is embedded as a Zariski-dense subgroup in $H:=\bar{G} / R(\bar{G})$ so that $H(\mathbb{R})$ is a semi-simple real linear algebraic group and is either of real rank 1 (cf. [14, 0.25]) or locally isomorphic to $\mathrm{SL}_{3}(\mathbb{R})$ or $\mathrm{SL}_{3}(\mathbb{C})$.

(2) Suppose that $R(G)$ is not of null entropy. Set $B:=\operatorname{Aut}(X)$. Then there is a $B$-(and hence $G$-) equivariant birational surjective morphism $X \rightarrow X^{\prime}$ such that $X^{\prime} \cong T / F$ for a finite group $F$ acting freely outside a finite set of an abelian variety $T$ of dimension three. Further, the action of $B$ on $X^{\prime}$ lifts to an action of a group $\widetilde{B}$ on $T$ such that $\widetilde{B} / F \cong B$.

We now prove Theorem 4.2. Note that $\iota(G)$ is contained in $\bar{G}(\mathbb{Q})$, and is Zariski-dense in $\bar{G}$. As in Lemma 2.5. replacing $G$ by a suitable finite-index subgroup, we may assume $\bar{G}$ is connected. Set $R:=R(G)$. The $\iota: G \rightarrow \bar{G}$ above induces an injective homomorphism:

$$
\gamma: G / R \rightarrow H:=\bar{G} / R(\bar{G})
$$

Indeed, $\gamma$ is defined over $\mathbb{Q}$ (cf. [14, 0.11]). Of course, $H$ is semi-simple. $R \mid \mathrm{NS}_{\mathbb{C}}(X)$ is solvable, being embedded in the solvable group $R(\bar{G})$. 
Lemma 4.3. Up to finite index, $H(\mathbb{R})$ is either semi-simple and of real rank one, or locally isomorphic to $\mathrm{SL}_{3}(\mathbb{R})$ or $\mathrm{SL}_{3}(\mathbb{C})$.

Proof. Let $S$ be a Levi subgroup of $\bar{G}$ such that $\bar{G}=R(\bar{G}) S$. Our $S$ can be chosen to be defined over $\mathbb{Q}$ and the induced composite homomorphism $S \rightarrow \bar{G} \rightarrow H$ is a $\mathbb{Q}$-isogeny (cf. [4, Proof of Proposition 11.23]). Now the argument in [7, Theorem 5.1, Proposition $5.2]$ for the action $S(\mathbb{R}) \mid \mathrm{NS}_{\mathbb{R}}(X)(\mathbb{Q}$-isogeny to $H(\mathbb{R})$ ) implies that either $H(\mathbb{R})$ is of real rank $\leq 1$, or $H(\mathbb{R})$ is of real rank $\geq 2$ and is locally isomorphic to $\mathrm{SL}_{3}(\mathbb{R})$ or $\mathrm{SL}_{3}(\mathbb{C})$. In fact, our $S(\mathbb{R})$ and indeed even the larger group $\bar{G}(\mathbb{R})$ already act on $\mathrm{NS}_{\mathbb{R}}(X)$ as the extension of the geometrically induced action of the Zariski-dense subgroup $G \mid \mathrm{NS}_{\mathbb{R}}(X)$ of $\bar{G}(\mathbb{R})$, so we do not need Margulis' condition on $G \mid \mathrm{NS}_{\mathbb{R}}(X)$ there, for the extension of the action. To be precise, one main purpose of the extra assumption in [7] on the rank of a lattice (acting on $X$ ) of a semi-simple real Lie group is to extend the action of the lattice on cohomology groups of $X$ to an action of the real Lie group.

If $H$ has real rank $\operatorname{rk}_{\mathbb{R}} H=0$, then $H(\mathbb{R})$ is compact. The image of $G$ in $H(\mathbb{R})$ is contained in an arithmetic subgroup of $H$, hence discrete and finite. This image is Zariski-dense in $H(\mathbb{R})$. Thus $H(\mathbb{R})$ is a finite group and hence $G$ is a finite extension of $R$, so $G \mid \mathrm{NS}_{\mathbb{C}}(X)$ is virtually solvable, which contradicts the assumption. Thus, $\operatorname{rk}_{\mathbb{R}} H$ is at least one and the lemma is proved.

We return to the proof of Theorem 4.2. Suppose that $R$ is of null entropy. As mentioned in the proof of Theorem 2.2 (cf. [16, Proposition 2.2]) the set

$$
U(R):=\left\{g \in R ; g^{*} \mid \mathrm{NS}_{\mathbb{C}}(X) \text { is unipotent }\right\}
$$

is a finite-index subgroup of $R$. So Theorem 4.2(1) occurs by the lemma above.

Hence we may assume that $R$ is not of null entropy. We will deduce Theorem 4.2(2). Take $R_{1} \leq R$ a finite-index subgroup such that $R_{1} \mid \mathrm{NS}_{\mathbb{C}}(X)$ has connected Zariski-closure in $\operatorname{GL}\left(\mathrm{NS}_{\mathbb{C}}(X)\right)$ (and is solvable). Thus $R_{1} / N\left(R_{1}\right) \cong \mathbb{Z}^{\oplus r}$ for some $1 \leq r \leq \operatorname{dim} X-1=2$ by [23, Theorem 1.2]. If $r=2$, then Theorem 4.2(2) holds by Theorem 4.1.

Thus we may assume $r=1$, i.e., $\langle\bar{h}\rangle=R_{1} / N\left(R_{1}\right) \cong \mathbb{Z}$ with $h \in R_{1}$ of positive entropy.

Lemma 4.4. $K_{X} \sim_{\mathbb{Q}} 0$.

Proof. Suppose the contrary that the lemma is false. By the 3-dimensional minimal model program and abundance theorem (cf. [12, 3.13]), the Kodaira dimension $\kappa(X)$ is positive, and $\left|m K_{X}\right|$ (for some $m>0$ ) defines a holomorphic map $\psi: X \rightarrow Y$ with connected fibres and $\operatorname{dim} Y=\kappa(X)$. The induced action of $G$ on $Y$ is trivial if $G$ is replaced by a suitable finite-index subgroup (cf. [20, Theorem 14.10]). Hence $G$ acts faithfully on a general fibre $S$ of $\psi$. Under the identification $G \cong G \mid S$, we have $N(G \mid S)=N(G) \mid S$ 
(cf. [24, 2.1(11) Remark]). Since $G \neq N(G)$, our $G \mid S$ is not of null entropy. Hence $\operatorname{dim} S \geq 2$. Also $\operatorname{dim} S=\operatorname{dim} X-\operatorname{dim} Y \leq \operatorname{dim} X-1=2$. Thus $\operatorname{dim} S=2$.

In the notation above, $\left(R_{1} \mid S\right) / N\left(R_{1} \mid S\right) \cong \mathbb{Z}$. Hence the restrictions of $R_{1}$ and $R$ on $\mathrm{NS}_{\mathbb{C}}(S)$ are virtually solvable (cf. Theorem 2.2 or 2.3 ). Lemma 2.8 is applicable to $R|S \triangleleft G| S$ since the conditions in Lemma 2.8 (2) (for surfaces) follow from the condition in Lemma 2.8 (1). Indeed, by the cone theorem of Lie-Kolchin type [23, Theorem 1.6], $R_{1}$ (replaced by a finite-index subgroup) has a common nonzero nef eigenvector $L_{1}$; thus the class $h^{*} L_{1}$ is parallel to $L_{1}$; after switching $h$ with $h^{-1}$ if necessary, [22, Lemma 2.12] implies that $h^{*} L_{1}=d_{1}(h) L_{1}$, and also the second condition in Lemma 2.8 (2). So, by Lemma 2.8, replacing $G$ by its subgroup of index $\leq 2, L_{h}$ gives rise to a character $\chi: G \mid S \rightarrow\left(\mathbb{R}_{>0}, \times\right)$ and that (for surfaces) $\operatorname{Ker}(\chi)=N(G \mid S)$. So the null set $N(G \mid S)$ is a subgroup and $G / N(G)=(G \mid S) / N(G \mid S) \cong \operatorname{Im} \chi$, an abelian group. Hence $G \mid L_{\mathbb{C}}$ is virtually solvable (cf. Theorem 2.2 or 2.3), contradicting the assumption.

In notation of 2.1, there exist two nonzero nef divisors $L_{h}, L_{h^{-1}}$ (which will be fixed) such that $\left(h^{ \pm 1}\right)^{*} L_{h^{ \pm 1}}=d_{1}\left(h^{ \pm 1}\right) L_{h^{ \pm 1}}$ with $d_{1}\left(h^{ \pm 1}\right)>1$.

Claim 4.5. Suppose there are a nef $\mathbb{R}$-divisor $M$, a real number $\lambda \neq 1$ and an integer $s \neq 0$ such that $\left(h^{s}\right)^{*} M=\lambda M$ and $M$ is not parallel to $L_{h^{ \pm 1}}$. Then Theorem 4.2(2) holds.

Proof. Note that $\left(h^{s}\right)^{*} L_{h}=d_{1}(h)^{s} L_{h},\left(h^{s}\right)^{*} L_{h^{-1}}=d_{1}\left(h^{-1}\right)^{-s} L_{h^{-1}}$, and $\left(h^{s}\right)^{*} M=\lambda^{s} M$. Rewriting $h^{s}$ as $h$, we may assume $s=1$. We have $M . c_{2}(X)=h^{*}\left(M \cdot c_{2}(X)\right)=$ $h^{*} M . h^{*} c_{2}(X)=\lambda M . c_{2}(X)$. Hence $M . c_{2}(X)=0$ for $\lambda \neq 1$. Similarly, $L_{h^{ \pm 1}} . c_{2}(X)=$ 0. By the assumption, M. $L_{h^{ \pm 1}} \neq 0$ (cf. [8, Corollary 3.2]). Thus, since $M, L_{h}, L_{h^{-1}}$ are nef eigenvectors of $h^{*}$ corresponding to eigenvalues $\lambda, d_{1}(h), 1 / d_{1}\left(h^{-1}\right)$ and since $d_{1}(h) \neq 1 / d_{1}\left(h^{-1}\right)$, [8, Lemma 4.4] implies that the product of these three nef divisors is nonzero and hence the sum of these three is a nef and big divisor, perpendicular to $c_{2}(X)$. So Theorem 4.2(2) holds true, by Theorem 4.1(1) and Lemma 4.4.

We return to the proof of Theorem 4.2. As proved above, the closed cone $\operatorname{Nef}(X) \cap$ $c_{2}(X)^{\perp}=\left\{M \in \operatorname{Nef}(X) \mid M . c_{2}(X)=0\right\}$ contains $L_{h^{ \pm 1}}$. Since $R_{1} \mid \mathrm{NS}_{\mathbb{C}}(X)$ is solvable, the cone theorem of Lie-Kolchin type (cf. e.g. [23, Theorem 2.6]) implies that the above closed cone contains a nonzero common nef divisor $L_{1}$ (with $L_{1} \cdot c_{2}(X)=0$ ) of $R_{1}$, after $R_{1}$ is replaced by a finite-index subgroup. Write $g^{*} L_{1}=\chi(g) L_{1}$ and consider the homomorphism

$$
\varphi: R_{1} \rightarrow(\mathbb{R},+), \quad g \mapsto \log \chi(g) .
$$

Clearly, $N\left(R_{1}\right) \leq \operatorname{Ker}(\varphi)$ (cf. 2.1). If $g \in \operatorname{Ker}(\varphi) \backslash N\left(R_{1}\right)$, then the product of the three nef eigenvectors $L_{1}, L_{g^{ \pm 1}}$ (corresponding to different eigenvalues $1, d_{1}(g) \neq 1 / d_{1}\left(g^{-1}\right)$ of $\left.g^{*}\right)$ is nonzero by [8, Lemma 4.4] and hence the sum of these three vectors is a nef and 
big $\mathbb{R}$-divisor class perpendicular to $c_{2}(X)$. Thus, by Lemma 4.4, we can apply Theorem 4.1(1) to conclude Theorem 4.2(2).

Therefore, we may assume that $\operatorname{Ker}(\varphi)=N\left(R_{1}\right)$. In particular, $\chi(h) \neq 1$, where $h^{*} L_{1}=\chi(h) L_{1}$. Possibly switching $h$ with $h^{-1}$, we may assume that $\chi(h)>1$. By Claim 4.5, we may assume that $L_{1}=L_{h}$ which is a common eigenvector of $R_{1}$. Thus the condition (1) of Lemma 2.8 is satisfied while the condition (2) can be assumed in view of Claim 4.5, So, by Lemma 2.8, replacing $G$ by its subgroup of index $\leq 2$, we may assume that $G$ fixes $L_{h}$ up to scalars. Write $g^{*} L_{h}=\chi^{\prime}(g) L_{h}$. Let

$$
\psi: G \rightarrow(\mathbb{R},+), \quad g \mapsto \log \chi^{\prime}(g)
$$

so that $G / \operatorname{Ker}(\psi)$ is mapped to an abelian subgroup of $(\mathbb{R},+)$. If $\operatorname{Ker}(\psi) \neq N(G)$, then as in the case of $\operatorname{Ker}(\varphi) \neq N(G)$ above, we take $g \in \operatorname{Ker}(\psi) \backslash N(G)$, so $c_{2}(X)$ is perpendicular to the nef and big divisor $L_{1}+L_{g}+L_{g^{-1}}$ and hence Theorem $4.2(2)$ occurs.

Thus we may assume that $N(G)=\operatorname{Ker}(\psi)$ which is hence a subgroup of $G$. Since $G / N(G) \cong \operatorname{Im} \psi$ is abelian, $G \mid \mathrm{NS}_{\mathbb{C}}(X)$ is virtually solvable by Theorem 2.2 or 2.3 , contradicting the assumption. The proof of Theorem 4.2 is completed.

\subsection{Proof of Theorem 1.1}

We may assume that Theorem $4.2(2)$ occurs and use the notation there. Let $\widetilde{G}$ be the lifting to $T$ of $G \mid X^{\prime}$ with $\widetilde{G} / F=G \mid X^{\prime}$. As sets (and set of left cosets), we have equalities $N(\widetilde{G}) / F=N\left(G \mid X^{\prime}\right)=N(G) \mid X^{\prime}$; so $N(G) \leq G$ if and only if $N(\widetilde{G}) \leq \widetilde{G}$, and if this is the case $\widetilde{G} / N(\widetilde{G}) \cong G / N(G)$ (cf. [24, Lemma 2.6]). Thus, by Theorem 2.3(3), as on $X$, neither $G \mid \mathrm{NS}_{\mathbb{C}}\left(X^{\prime}\right)$ nor $\widetilde{G} \mid \mathrm{NS}_{\mathbb{C}}(T)$ is virtually solvable. By the same reasoning, the lifting to $T$ of $R(G) \mid X^{\prime}$ has virtually solvable action on $\operatorname{NS}_{\mathbb{C}}(T)$, is normal in $\widetilde{G}$ and is not of null entropy. $R(\widetilde{G})$ contains this lifting up to finite index, so it is not of null entropy. Hence we may assume that $X=T$, a complex torus.

Let $\hat{G} \leq \mathrm{GL}\left(H^{0}\left(X, \Omega_{X}^{1}\right)^{\vee}\right)=\mathrm{GL}_{3}(\mathbb{C})$ be the Zariski-closure of the action $G \mid H^{0}\left(X, \Omega_{X}^{1}\right)^{\vee}$. Since every $g \in G$ acts on $H^{1}(X, \mathbb{Z})$ invertibly, its matrix representation has determinant \pm 1 ; note also $H^{1}(X, \mathbb{C})=H^{0}\left(X, \Omega_{X}^{1}\right) \oplus H^{0}\left(X, \Omega_{X}^{1}\right)^{\vee}$; hence we may assume that $\hat{G}$ is contained in $\mathrm{SL}_{3}(\mathbb{C})$ and connected, after $G$ is replaced by a finite-index subgroup.

Since $H^{*}(X, \mathbb{C}):=\oplus_{i \geq 0} H^{i}(X, \mathbb{C})$ is generated by wedge products of $H^{0}\left(X, \Omega_{X}^{1}\right)$ and its conjugate, the null set $N(G)$ is equal to $\left\{g \in G ; g \mid H^{0}\left(X, \Omega_{X}^{1}\right)\right.$ is of null entropy $\}$. Let

$$
R:=G \cap R(\hat{G}) \triangleleft G, \quad U:=G \cap U(\hat{G}) \triangleleft G .
$$

Then $R(\hat{G}) \mid H^{*}(X, \mathbb{C})$ and hence $R \mid H^{*}(X, \mathbb{C})$ are solvable. By Theorem 2.3 , $R$ has a finite-index subgroup $R_{1}$ such that

$$
\mathbb{Z}^{\oplus r} \cong R_{1} / N\left(R_{1}\right) \leq R / N(R)
$$


Also $|N(R): U|<\infty$ (cf. Theorem 2.2). Thus $R / U$ contains a copy of $\mathbb{Z}^{\oplus r}$ as a subgroup of finite index (cf. Lemma 2.4). Consider the natural embedding

$$
G / U \rightarrow J:=\hat{G} / U(\hat{G})
$$

into the reductive group $J$ of real rank $\leq \operatorname{rk}_{\mathbb{R}} \mathrm{SL}_{3}(\mathbb{C})=2$.

If $r \geq 2$, then $\operatorname{rk}_{\mathbb{R}} J=2$; the Zariski-closure of $R / U \subset J$ contains a copy of $\mathbb{Z}^{\oplus 2}$ and hence a maximal torus of $J$, and is normal in $J$, so this closure equals $J$. Thus $J$ (like $R / U)$ and hence the actions of $G / U$ and $G$ on $H^{*}(X, \mathbb{C})$ are all solvable, contradicting the assumption.

Consider the case $r=0$, i.e., $R \subseteq N(G)$. This contradicts the extra assumption that $R(G)$ is not of null entropy and the assertion $(*): R(G)$ equals $R$ up to finite index. Indeed, as in the proof of Lemma 2.6, $G \mid \mathrm{NS}_{\mathbb{C}}(X)=G / K$ with $\left|K: G \cap \operatorname{Aut}_{0}(X)\right|<\infty$, while $G \mid H^{0}\left(X, \Omega_{X}^{1}\right)^{\vee}=G /\left(G \cap \operatorname{Aut}_{0}(X)\right)$. Hence $G \mid \mathrm{NS}_{\mathbb{C}}(X)$ equals $G \mid H^{0}\left(X, \Omega_{X}^{1}\right)^{\vee}$ modulo a finite group. Now the assertion $\left(^{*}\right)$ follows from the definitions of $R(G)$ and $R$.

Finally, assume $r=1$, i.e., $R_{1} / N\left(R_{1}\right) \cong \mathbb{Z}$. For any $g_{1} \in G$, the group $G_{1}:=\left\langle g_{1}, R\right\rangle$ (replaced by its finite-index subgroup) has $G_{1} \mid H^{*}(X, \mathbb{C})$ solvable and $G_{1} / N\left(G_{1}\right) \cong \mathbb{Z}^{\oplus s}$ (cf. Theorem 2.3). We claim that $s \geq 2$ for some $g_{1}$. If the claim is false, then for any $g_{1} \in G$, we have $s \leq 1$ and hence $g_{1}^{a}=h^{b} n$ for some $a \geq 1, n \in N(G)$, where $\langle\bar{h}\rangle=$ $R_{1} / N\left(R_{1}\right) \leq G_{1} / N\left(G_{1}\right)$. Thus $g_{1}(\bmod R)$ has a positive power acting as a unipotent element on $H^{0}\left(X, \Omega_{X}^{1}\right)^{\vee}$ because the same is true for $n \in N(G)$. So the subgroup $G / R$ of an arithmetic subgroup of $\hat{G} / R(\hat{G})$ (defined over $\mathbb{Q}$; cf. [14, ChI; 0.11]) has a unipotent group $U(G / R)$ as its subgroup of finite index, by Burnside's theorem as in [16, Proposition 2.2] or Theorem 2.2. Thus its Zariski-closure $\hat{G} / R(\hat{G})$ is both unipotent and semi-simple and hence trivial. So $G \mid H^{*}(X, \mathbb{C})$ is solvable, contradicting the assumption.

Thus the claim is true and hence some $G_{1}:=\left\langle g_{1}, R\right\rangle$ has $G_{1} / N\left(G_{1}\right) \cong \mathbb{Z}^{\oplus s}$ for some $s \geq 2$. So by [26, Paragraph before $\S 2.8], U\left(G_{1}\right)$ and hence $N\left(G_{1}\right)$ and $N(R)$ act as finite groups on $H^{0}\left(X, \Omega_{X}^{1}\right)$ and also on $H^{*}(X, \mathbb{C}$ ) (cf. Proof of Theorem 2.2). Thus, when restricted on $H^{0}\left(X, \Omega_{X}^{1}\right)^{\vee}$, our $R$ (containing a finite-index subgroup $R_{1}$ with $R_{1} / N\left(R_{1}\right) \cong$ $\mathbb{Z})$ is virtually infinite cyclic and normalized by $G$, so it is contained in the centre of $G \mid H^{0}\left(X, \Omega_{X}^{1}\right)^{\vee}$ and of $\hat{G}$, by replacing $G$ by a finite-index subgroup and considering the conjugate action on the derived series of $R$.

Now we follow referee's suggestion. Take an element $h \in R \backslash N(R)$. If $h \mid H^{0}\left(X, \Omega_{X}^{1}\right)^{\vee}$ $\in \mathrm{SL}_{3}(\mathbb{C})$ has three distinct eigenvectors, then $h$ and all elements of $G$ are simultaneously diagonalizable and hence $G \mid H^{*}(X, \mathbb{C})$ is abelian, contradicting the assumption. 
Therefore, relative to a suitable basis $B$ of $H^{0}\left(X, \Omega_{X}^{1}\right)^{\vee}$, our $h \mid H^{0}\left(X, \Omega_{X}^{1}\right)^{\vee}$ is in one of the Jordan canonical forms

$$
\operatorname{block} \operatorname{diag}\left[\alpha^{-2}, J_{2}(\alpha)\right], \quad \operatorname{diag}\left[\alpha^{-2}, \alpha, \alpha\right]
$$

and the matrix representation $g \mid H^{0}\left(X, \Omega_{X}^{1}\right)^{\vee}=\left(a_{i j}\right)$ of every $g \in G$ is especially upper triangular. Consider the projection

$$
\tau: G \rightarrow \mathbb{C}^{*}, \quad g \mapsto a_{11}
$$

If $\operatorname{Ker} \tau \subseteq N(G)$, then the actions of $\operatorname{Ker} \tau$ and hence of $(\operatorname{Ker} \tau) R$ on $H^{0}\left(X, \Omega_{X}^{1}\right)^{\vee}$ are virtually solvable (cf. Theorem 2.2) , so is that of $G$, because $G /((\operatorname{Ker} \tau) R$ ) is a quotient of the abelian group $\operatorname{Im} \tau$. This contradicts the assumption.

Thus, we can take $g_{1} \in \operatorname{Ker} \tau \backslash N(G)$. Then $g_{1} \mid H^{0}\left(X, \Omega_{X}^{1}\right)^{\vee}$ has 3 eigenvalues $1, \lambda^{ \pm 1}$ (with $|\lambda| \neq 1$ ); it has a unique (up to scalar) eigenvector $w \in H_{1}(X, \mathbb{Z})$ (= the lattice $\Lambda$ of the torus $X=\mathbb{C}^{3} / \Lambda$ ) corresponding to the eigenvalue $1 \in \mathbb{Q}$ and is proportional to the column vector $(1,0,0)^{t}$ (in basis $B$ ). Now $h$ or $h^{-1}$ takes $w$ to $\alpha^{-2} w$ with $\left|\alpha^{-2}\right|<1$. This contradicts the fact that $h(\Lambda)=\Lambda$ which is discrete in $\mathbb{C}^{3}=\mathbb{R}^{6}$. Theorem 1.1 is proved.

\section{REFERENCES}

[1] A. Beauville, Some remarks on Kähler manifolds with $c_{1}=0$, Classification of Algebraic and Analytic Manifolds (Katata, 1982, ed. K. Ueno), Progr. Math. 39, Birkhäuser, 1983, pp. 1-26.

[2] C. Birkar, P. Cascini, C. D. Hacon and J. McKernan, Existence of minimal models for varieties of log general type, J. Amer. Math. Soc. 23 (2010) 405-468.

[3] G. Birkhoff, Linear transformations with invariant cones, Amer. Math. Monthly 74 (1967), 274-276.

[4] A. Borel, Linear algebraic groups, 2nd ed. Graduate Texts in Math., 126, Springer-Verlag, 1991.

[5] S. Cantat, Dynamique des automorphismes des surfaces projectives complexes, C. R. Acad. Sci. Paris Ser. I Math. 328(1999), no. 10, 901-906.

[6] S. Cantat, Sur les groupes de transformations birationnelles des surfaces, Ann. of Math. 174 (2011), 299-340.

[7] S. Cantat and A. Zeghib, Holomorphic actions of higher rank lattices in dimension three, Preprint.

[8] T.-C. Dinh and N. Sibony, Groupes commutatifs d'automorphismes d'une variété kählerienne compacte, Duke Math. J. 123 (2004), no. 2, 311-328.

[9] B. Fu and D. -Q. Zhang, in preparation.

[10] A. Fujiki, On automorphism groups of compact Kähler manifolds, Invent. Math. 44 (1978), no. 3, $225-258$.

[11] M. Gromov, On the entropy of holomorphic maps, Enseign. Math. (2) 49 (2003), no. 3-4, 217-235. (first appeared as SUNY preprint in 1977).

[12] J. Kollár and S. Mori, Birational geometry of algebraic varieties, Cambridge Tracts in Math. 134, Cambridge Univ. Press, 1998.

[13] D. I. Lieberman, Compactness of the Chow scheme: applications to automorphisms and deformations of Kähler manifolds, pp. 140-186, Lecture Notes in Math. 670, Springer, 1978. 
[14] G. A. Margulis, Discrete subgroups of semisimple Lie groups, Springer-Verlag, 1991.

[15] M. Miyanishi, Algebraic methods in the theory of algebraic threefolds - surrounding the works of Iskovskikh, Mori and Sarkisov, 69-99, Adv. Stud. Pure Math. 1, North-Holland, Amsterdam, 1983.

[16] K. Oguiso, Automorphisms of hyperkähler manifolds in the view of topological entropy, Algebraic geometry, 173-185, Contemp. Math. 422, Amer. Math. Soc., Providence, RI, 2007.

[17] K. Oguiso and J. Sakurai, Calabi-Yau threefolds of quotient type, Asian J. Math. 5 (2001), 43-77.

[18] N. I. Shepherd-Barron and P. M. H. Wilson, Singular threefolds with numerically trivial first and second Chern classes, J. Algebraic Geom. 3 (1994), no. 2, 265-281.

[19] J. Tits, Free subgroups in linear groups, J. Algebra 20 (1972), 250-270.

[20] K. Ueno, Classification theory of algebraic varieties and compact complex spaces, Notes written in collaboration with P. Cherenack, Lecture Notes in Mathematics, Vol. 439, Springer-Verlag, BerlinNew York, 1975.

[21] Y. Yomdin, Volume growth and entropy, Israel J. Math. 57 (1987), no. 3, 285-300.

[22] D. -Q. Zhang, Automorphism groups and aniti-pluricanonical curves, Math. Res. Lett. 15 (2008), no. $1,163-183$.

[23] D. -Q. Zhang, A theorem of Tits type for compact Kähler manifolds, Invent. Math. 176 (2009), no. $3,449-459$.

[24] D. -Q. Zhang, Dynamics of automorphisms on projective complex manifolds, J. Differential Geom. 82 (2009), no. 3, 691-722.

[25] D. -Q. Zhang, Polarized endomorphisms of uniruled varieties (with an Appendix by Y. Fujimoto and N. Nakayama), Compos. Math. 146 (2010), no. 1, 145-168.

[26] D. -Q. Zhang, Automorphism groups of positive entropy on minimal projective varieties, Adv. Math. 225 (2010), no. 5, 2332-2340.

\section{Department of Mathematics}

University of Nancy 1, BP 239, F-54506, Vandoeuvre-les-Nancy, Cedex, France

E-mail address: Frederic.Campana@iecn.u-nancy.fr

\section{Department of Mathematics}

National University of Singapore, 10 Lower Kent Ridge Road, Singapore 119076

E-mail address: matwf@nus.edu.sg

Department of Mathematics

National University of Singapore, 10 Lower Kent Ridge Road, Singapore 119076

E-mail address: matzdq@nus.edu.sg 